\title{
A NOVEL SOLUTION FOR GROUND REACTION CURVE OF TUNNELS IN ELASTOPLASTIC STRAIN SOFTENING ROCK MASSES
}

\author{
Ali GHORBANI, Hadi HASANZADEHSHOOIILI \\ Department of Civil Engineering, Faculty of Engineering, The University of Guilan, Rasht, Guilan, Iran
}

Received 27 Jul 2016; accepted 05 Dec 2016

\begin{abstract}
Ground Reaction Curve (GRC) is one of the most important elements of convergence-confinement method generally used to design tunnels. Realistic presentation of GRC is usually assessed based on the advanced rock strength criteria, also, rock mass behavior (including plasticity and softening treatments). Since taking these parameters into account is not simply possible for practitioners and needs complicated coupled theoretical-numerical solutions, this paper presents a simple novel approach based on Evolutionary Polynomial Regression to determine GRC of rock masses obeying both Mohr-Coulomb and Hoek-Brown criteria and strain softening behaviors. The proposed models accurately present support pressures based on radial displacement, rock mass strength and softening parameter (determination coefficient of $97.98 \%$ and $94.2 \%$ respectively for Mohr-Coulomb and Hoek-Brown strain softening materials). The accuracy of the proposed equations are approved through comparing the EPR developed GRCs with the ground reaction curves available in the literature. Besides, the sensitivity analysis is carried out and in-situ stress, residual Hoek-Brown's $m$ constant and residual dilation angle are introduced as parameters with the most influence on the support pressure in Hoek-Brown and peak and residual geological strength index are the most affective parameters on the support pressure of tunnels in the strain softening Mohr-Coulomb rock mass.
\end{abstract}

Keywords: ground reaction curve, circular tunnel, rock mass, Mohr-Coulomb, Hoek-Brown, strain softening, evolutionary polynomial regression, sensitivity analysis.

\section{Introduction}

Tunneling projects consist of different phases including pre-construction studies, risk assessment, cost evaluation, design phase, construction, monitoring (sometimes some of the mentioned phases will be merged when performance based design is the selected design method) (Sayadi et al. 2012; Hasanzadehshooiili et al. 2012a, 2012b; Ghorbani et al. 2013; Zavadskas et al. 2015; Zhang et al. 2016). One of the major issues in design of tunnels is the loads carried by tunnel linings (Ghorbani et al. 2013). Nevertheless, there are some main problems such as uncertainties in rock nature, variation of ground condition, ground deformation induced by redistribution of in-situ stresses and difficult computational procedure, which makes it difficult to accurately and easily obtain the pressure on and sustained by internal support of rock circular tunnels (Hasanzadehshooiili et al. 2012b). In this regard, it is common among practitioners to design tunnel structures more conservatively. Design of support system of tunnels, due to the problem complexities, has always been one of the most important and complicated problems in the tunnel engineering field (Hasanzadehshooiili et al. 2012a). Since rock mass properties are not the only parameters controlling rocks' stress-strain behavior, special attentions should be paid to the support type and the time of support installation, which makes it an interaction rock-support problem. Solutions proposed for the rock- support interaction problem can be categorized in the following ones: closed-form analytical methods, empirical methods, numerical methods (e.g. finite element, finite difference, distinct element and boundary element methods). Among the mentioned methods, convergenceconfinement method is one of the most in demanding design methods widely used by tunnel and rock mechanics engineers. This method is founded based on three fundamental elements consisting longitudinal deformation profile (LDP), support characteristic curve (SCC) and ground reaction curve (GRC) (Brown et al. 1983).

As a simple explanation, the radial support pressure, $P_{i}$, required at a particular point on the boundary of excavation to limit the radial convergence to $U_{i}$, is represented by the ground reaction curve (Fig. 1), where $U_{i}$ is radial tunnel displacement and $P_{i}$ represents corresponding internal support pressure of tunnels. 


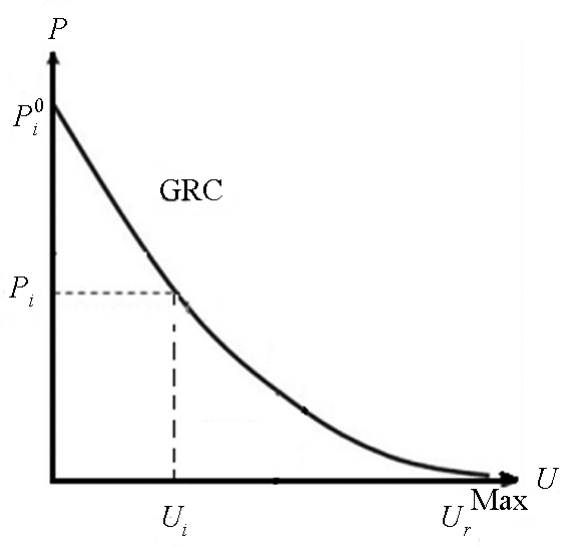

Fig. 1. Schematic view of an idealized GRC for circular tunnel

Considering the parameters affecting on the problem, constituting elements of convergence-confinement method, especially ground reaction curve, are studied based on different theoretical, numerical or coupled theoretical-numerical methods. In development of ground reaction (response) curve, changes in the support pressures due to the tunneling is tracked near the tunnel face and the concerning radial tunnel displacement-support pressure curve is reported as the GRC, which is conjunctly used with SCC to gain the optimum time of installation of the support system.

\section{Literature survey}

Ground reaction curves are usually developed based on elastic-brittle, elastic perfect plastic and elastic brittle plastic rock mass behavior considering strain softening. Fully theoretical schemes only present solutions to the elastic brittle or elastic perfect plastic rock mass behaviors (Sharan 2003, 2005, 2008). Besides, in such cases, simple strength criteria, post peak behaviors or some main simplifications are often assumed to idealize the problem. Hence, since it is not possible to find the analytical closed form solutions to the more realistic rock mass behavior, complicated strain-softening material behavior, the GRC is often calculated using coupled theoreticalnumerical approaches (Alonso et al. 2003; Guan et al. 2007; Lee, Pietruszczak 2008; Park et al. 2008; Alejano et al. 2009, 2010; Wang, Yin 2011; Zhang et al. 2012; Zareifard, Fahimifar 2012; González-Cao et al. 2013). Figure 2 presents different post peak behaviors of rock mass. In this figure, GSI represents geological strength index originally proposed by Hoek and Brown (1997).

As it can be seen in Figure 2, elastic brittle plastic and elastic perfectly plastic behaviors can be referred as two special cases for the strain softening model (Alejano et al. 2009). In strain softening models, material's behavior is commonly described using the peak and residual strength parameters for the materials. Indeed, it is assumed that the material follows a gradual transition from

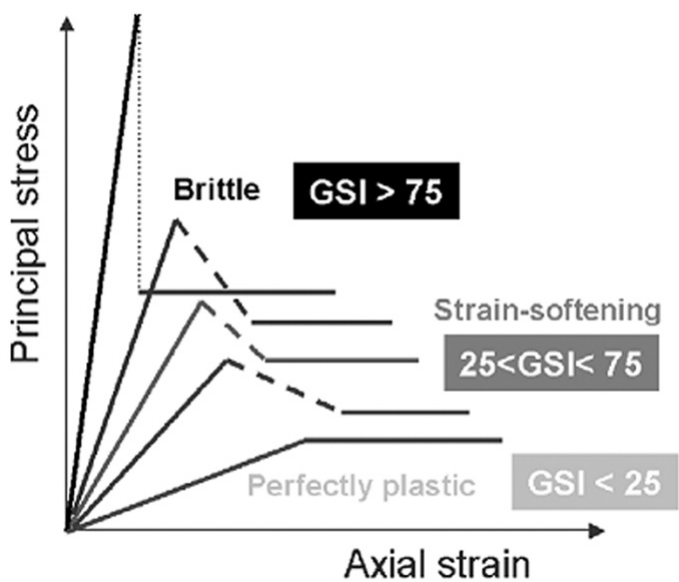

Fig. 2. Different post peak rock mass behaviors (Hoek, Brown 1997)

peak to the residual strength and the material's strength parameters are linearly decreased from their peak values to the corresponding residual ones. Slope of the transition line and the initiation of residual behavior are defined based on plasticity calculations (in a direct or an indirect way).

The proposed relations between materials peak and residual strength parameters for Hoek-Brown failure criterion are presented in Eqns (1)-(2) (Alejano et al. 2010):

$$
\begin{aligned}
& m(\eta)=\left\{\begin{array}{lr}
m^{\text {peak }}-\frac{m^{\text {peak }}-m^{\text {res }}}{\eta^{*}} \eta & 0<\eta<\eta^{*} \\
m^{\text {res }} & \eta \geq \eta^{*}
\end{array}\right\} ; \\
& s(\eta)=\left\{\begin{array}{ll}
s^{\text {peak }}-\frac{s^{\text {peak }}-s^{\text {res }}}{\eta^{*}} \eta & 0<\eta<\eta^{*} \\
s^{\text {res }} & \eta \geq \eta^{*}
\end{array}\right\},
\end{aligned}
$$

where $m^{\text {peak }}$ and $s^{\text {peak }}$ present peak strength HoekBrown parameters and $m^{\text {res }}$ and $s^{\text {res }}$ are its residual parameters. Also, $\eta^{*}$ is controlling softening parameter, which can be calculated in different ways. Hence, in order to derive GRC curve for a strain softening rock mass, it is essential to calculate strain softening parameter and to solve the governing differential equations simultaneously (equilibrium, compatibility and consistency equations along with plasticity rules and assumptions). Some of the GRC problems have been recently solved for different rock strength criteria showing different plasticity treatments and softening behaviors (Alonso et al. 2003; Mitaim, Detourney 2005; Xu, Yu 2006; Guan et al. 2007; Lee, Pietruszczak 2008; Fahimifar, Ranjbarnia 2009; Alejano et al. 2009, 2010; Osgoui 2009; Zhang et al. 2010; Zareifard, Fahimifar 2012; Zhang et al. 2012; GonzálezCao et al. 2013; Park 2014). Nevertheless, there is not still a general solution for some nonlinear failure criteria and softening behaviors for variable and stress dependent 
dilatancies under different loading conditions. Besides, using such computations is not so easy for practitioners and tunnel engineers. This is the main reason that advanced GRC solutions (strain softening) are not commonly used by tunnel engineers using simple calculations. In this regard, in this paper, two different rock strength criteria (Mohr-Coulomb and Hoek-Brown failure criteria) are considered and some of the available authenticated strain softening solutions are gathered and back calculated. Following, a simple novel approach to determine GRC curve of circular rock mass tunnels has been described and relations for both Mohr-Coulomb and Hoek-Brown failure criteria are presented. It is believed that these relationships can be efficiently used by practitioners to gain the GRC of Mohr-Coulomb and Hoek-Brown strain softening rock masses in preliminary design step of tunnels with some simple calculations.

\section{Problem statement}

As described in previous section, two different failure criteria, Mohr-Coulomb and Hoek-Brown strength criteria are considered and the available authenticated coupled theoretical-numerical solutions to the GRC of strain softening materials have been gathered. Following, general assumptions and governing rules of conventional solutions to the problem of ground reaction curve of a circular opening in an elastic-plastic strain softening rock mass are presented.

\subsection{Conventional GRC problem of circular tunnels in an elastoplastic strain softening rock mass}

In theses methods, material's strength criteria are assumed as the yield criteria and are used for the solution of material's governing differential equations. Also, plastic potential function, flow rule and treatment with strain softening parameters are other three important elements defining GRC. Figure 3 shows different regions formed around circular tunnels, elastic and plastic regions.

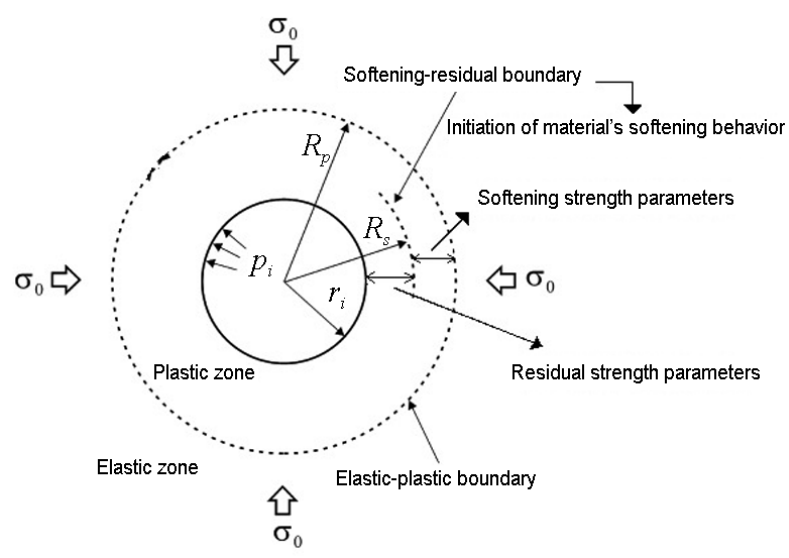

Fig. 3. General schematic regions around circular tunnel under hydrostatic stress field
Moreover, softening-residual boundary showing the boundary where material behavior is changed from softening to the residual is depicted. Indeed, beyond elastic-plastic boundary, material behaves perfectly elastic. Moving toward the tunnel boundary, plastic behavior initiates. In this zone, firstly, the peak strength parameters are mobilized and linearly softened to the residual values of strength parameters. There are different treatments to find the softening-residual boundary, also, the corresponding strength parameters (Lee, Pietruszczak 2008; Alejano et al. 2009, 2010). Some of them define a strain softening parameter (generally $\eta=\varepsilon_{1}{ }^{\text {plas }}-\varepsilon_{3}{ }^{\text {plas }}$ ) and an iterative procedure to cover the whole plastic region, calculating $\eta$ successively and comparing it with the critical strain softening parameter, $\eta^{*}$ (Lee, Pietruszczak 2008). The values of strain softening parameter beyond $\eta^{*}$ declares that the material's strength parameters are changed to their residual ones, while lower values show that they are still in softening zone and can be determined using Eqns (1) and (2). On the other hand, some other methods use $\sigma_{1}-\sigma_{3}, \sigma_{1}-\varepsilon_{1}$, the slope of $\varepsilon_{1}^{\text {plas }}-\varepsilon_{3}^{\text {plas }}$ curve (defined by coefficient of dilation) and the slope of transition line in strain softening region to obtain the values of $\varepsilon_{1}{ }^{\text {plas }}$ and $\varepsilon_{3}{ }^{\text {plas }}$ to calculate the value of $\eta$ and its critical value (Alejano et al. 2009, 2010). Such methods use an implicit scheme to solve the governing equilibrium and compatibility equations. The governing equilibrium equation in plane strain condition and cylindrical coordinate system for axial symmetry situation is as following (Park et al. 2008; Lee, Pietruszczak 2008):

$$
\frac{\partial \sigma_{r}}{\partial r}+\frac{\sigma_{r}-\sigma_{\theta}}{r}=0
$$

where $\sigma_{r}$ and $\sigma_{\theta}$, respectively, present radial and hoop stresses. Also, $r$ shows distance from the tunnel's centre. Moreover, relationship between radial and tangential plastic strains is defined using a suitable plastic potential function and applying associative/non-associative flow rule. The common general form of assumed plastic potential function, also, applied flow rule (commonly non-associative flow rule is considered) are respectively presented in Eqn (4) and Eqn (5) (Park et al. 2008; Lee, Pietruszczak 2008; Alejano et al. 2009, 2010; Zareifard, Fahimifar 2012):

$$
\begin{gathered}
G\left(\sigma_{r}, \sigma_{\theta}, \eta\right)=\sigma_{\theta}-k(\eta) \sigma_{r} \\
d \varepsilon_{r}^{\text {plas }}=-k(\eta) d \varepsilon_{\theta}^{\text {plas }}
\end{gathered}
$$

where $G$ is the plastic potential function and $d \varepsilon_{r}^{p l a s}$, $d \varepsilon_{\theta}^{p l a s}$ and $k(\eta)$ are radial increment of plastic strain, tangential increment of plastic strain and coefficient of dilation, respectively. Also, $\eta$ represents strain softening parameter.

Considering the described methods, GRC of circular tunnels are calculated using one of the iterative or implicit solutions. In this paper, in order to develop some 
new relationships for GRC, some of these solutions are gathered and further analyzed. In this regard, results of Park et al. (2008), Lee and Pietruszczak (2008), Alejano et al. (2009, 2010), Zareifard and Fahimifar (2012) presenting GRC curves for Mohr-Coulomb or Hoek-Brown criterion and satisfying strain softening situation are more focused and investigated.

\section{Data division for model development}

As explained earlier, GRC of the available sources along with their peak and residual strength parameters, also, critical strain softening parameters are gathered. Back calculating the obtained ground reaction curves, different $P_{i}$, internal support pressures of tunnels, and $U_{i}$, radial displacements of tunnel wall, values are digitized. Indeed, as an example and for a specific case, the entire peak, residual and strain softening parameters are assumed as input parameters. Besides, the points of GRC of the studied case are digitized. The values of displacements gained from the curves are also considered as the input parameter (each of the values of displacements makes a dataset, considering other strength and softening parameters) and the corresponding support pressures are used as the output parameters. Hence, it can be noted that each GRC constructs a few dozen of input-output datasets. Then, $P_{i}$, which is now considered as a function of tunnel wall radial displacement, rock mass strength and residual parameters and softening parameters, is then predicted using evolutionary polynomial regression modeling.

Regarding the fact that during data gathering phase, a wide range of material qualities and behaviors have been covered, from poor quality to hard rock, the resulting relations for $P_{i}$ (with a high degree of correlations with available solutions) can then be used for new rock strength and softening parameters in the early design stage of tunneling projects. The resulting $P_{i}-U_{i}$ relation can then be used as a first order estimation of GRC of tunnels in strain softening rock mass.

\subsection{GRC modeling based on the strain softening Mohr-Coulomb strength criterion}

In order to cover a wide range of material strength properties, three different cases studied by Alejano et al. (2009) are investigated and the concerning parameters are back calculated.

Tables $1-3$, respectively, present the materials' strength parameters and strain softening parameter for soft, medium and hard rock masses, where "peak" and "res" superscripts respectively represent peak and residual strength parameters and $G S I, \sigma_{c i}, \gamma, E, \vartheta, \psi$ and $\sigma_{0}$ are respectively geological strength index, uni-axial compressive strength, unit weight, Young's modulus, Poisson's ratio, dilation angle and in-situ stress of rock mass.

Also, $c, \varphi$ and $m_{i}$ are material constants. Besides, $r_{i}$ is the radius of tunnel and $\eta^{*}$ represents the strain softening parameter and is determined using
Table 1. Strength and softening parameters for soft rock mass used in Mohr-Coulomb model (Alejano et al. 2009)

\begin{tabular}{|c|c|c|}
\hline Parameters & Unit & Value \\
\hline$G S I^{\text {peak }}$ & - & 21.4 \\
\hline$G S I^{r e s}$ & - & 15.1 \\
\hline$\sigma_{c i}$ & $\mathrm{MPa}$ & 100 \\
\hline$m_{i}$ & - & 20 \\
\hline$\gamma$ & $\mathrm{kN} / \mathrm{m}^{3}$ & 26 \\
\hline$E$ & $\mathrm{GPa}$ & 1.1 \\
\hline$\vartheta$ & - & 0.3 \\
\hline$c^{p e a k}$ & $\mathrm{MPa}$ & 0.34 \\
\hline$\varphi^{p e a k}$ & ○ & 43 \\
\hline$c^{r e s}$ & $\mathrm{MPa}$ & 0.27 \\
\hline$\varphi^{r e s}$ & $\circ$ & 40 \\
\hline$\sigma_{0}$ & $\mathrm{MPa}$ & 10.4 \\
\hline$\psi$ & $\circ$ & 0 \\
\hline$\eta^{*}$ & - & 0.1394 \\
\hline
\end{tabular}

Table 2. Strength and softening parameters for medium rock mass used in Mohr-Coulomb model (Alejano et al. 2009)

\begin{tabular}{|c|c|c|}
\hline Parameters & Unit & Value \\
\hline$G S I^{\text {peak }}$ & - & 55 \\
\hline$G S I^{r e s}$ & - & 33 \\
\hline$\sigma_{c i}$ & $\mathrm{MPa}$ & 23 \\
\hline$m_{i}$ & - & 10 \\
\hline$\gamma$ & $\mathrm{kN} / \mathrm{m}^{3}$ & 26.7 \\
\hline$E$ & $\mathrm{GPa}$ & 3.837 \\
\hline$\vartheta$ & - & 0.25 \\
\hline$c^{p e a k}$ & $\mathrm{MPa}$ & 0.744 \\
\hline$\varphi^{p e a k}$ & 。 & 24.81 \\
\hline$c^{r e s}$ & $\mathrm{MPa}$ & 0.397 \\
\hline$\varphi^{r e s}$ & $\circ$ & 15.69 \\
\hline$\sigma_{0}$ & $\mathrm{MPa}$ & 12 \\
\hline$\psi$ & $\circ$ & 3.1 \\
\hline$\eta^{*}$ & - & 0.0929 \\
\hline
\end{tabular}


Table 3. Strength and softening parameters for hard rock mass used in Mohr-Coulomb model (Alejano et al. 2009)

\begin{tabular}{|c|c|c|}
\hline Parameters & Unit & Value \\
\hline$G S I^{\text {peak }}$ & - & 64.9 \\
\hline$G S I^{r e s}$ & - & 27.8 \\
\hline$\sigma_{c i}$ & $\mathrm{MPa}$ & 162 \\
\hline$m_{i}$ & - & 19 \\
\hline$\gamma$ & $\mathrm{kN} / \mathrm{m}^{3}$ & 26 \\
\hline$E$ & $\mathrm{GPa}$ & 24 \\
\hline$\vartheta$ & - & 0.25 \\
\hline$c^{\text {peak }}$ & $\mathrm{MPa}$ & 3.7 \\
\hline$\varphi^{\text {peak }}$ & $\circ$ & 57.8 \\
\hline$c^{r e s}$ & $\mathrm{MPa}$ & 0.96 \\
\hline$\varphi^{r e s}$ & $\circ$ & 51 \\
\hline$\sigma_{0}$ & $\mathrm{MPa}$ & 26 \\
\hline$\psi$ & $\circ$ & 14 \\
\hline$\eta^{*}$ & - & 0.0465 \\
\hline
\end{tabular}

Eqns (6)-(8) for soft, medium and hard rock, respectively (Alejano et al. 2009):

$$
\begin{aligned}
& \eta^{*}=6 \frac{\sigma_{1}^{\text {peak }}\left(\sigma_{3}\right)-\sigma_{1}^{\text {res }}\left(\sigma_{3}\right)}{E}\left(1+\frac{k_{\psi}}{2}\right) ; \\
& \eta^{*}=4 \frac{\sigma_{1}^{\text {peak }}\left(\sigma_{3}\right)-\sigma_{1}^{\text {res }}\left(\sigma_{3}\right)}{E}\left(1+\frac{k_{\psi}}{2}\right) ; \\
& \eta^{*}=2 \frac{\sigma_{1}^{\text {peak }}\left(\sigma_{3}\right)-\sigma_{1}^{\text {res }}\left(\sigma_{3}\right)}{E}\left(1+\frac{k_{\psi}}{2}\right),
\end{aligned}
$$

where $k_{\psi}$ is dilation parameter and is defined as the slope of $\sigma_{1}-\sigma_{3}$ diagram in Alejano et al. (2009) for the strain softening model with constant dilatancy and constant drop modulus. It is noteworthy to imply that the effect of depth of tunnel is also taken into account considering the far field intact stress, $\sigma_{0}$.

Regarding described cases and considering concerning strength and softening parameters, $P_{i}-U_{i}$ curve has been digitized and back calculated. Considering $U_{i}$ as an input parameter, different values of $P_{i}$ will be gained as a function of strength parameters, softening parameter and $U_{i}$. Regarding the described procedure, 168 independent data sets $P_{i}\left(r_{i}, G S I^{\text {peak }}, G S I^{\text {res }}, \sigma_{c i}, \gamma, E, \vartheta, c^{\text {peak }}\right.$, $\left.\varphi^{\text {peak }}, c^{r e s}, \varphi^{r e s}, m_{i}, \psi, \sigma_{0}, \eta^{*}, U_{i}\right)$ have been constructed ready for evolutionary polynomial regression modeling.

\subsection{GRC modeling based on the strain softening Hoek-Brown strength criterion}

The source for strength and softening parameters used to model GRC of Hoek-Brown strain softening rock mass (along with their treatment with strain softening parameter) are presented in Table 4, where $M$ represents drop modulus (slope of transition line in strain softening zone) and $E$ is material's Young's modulus.

Ground reaction curves developed using the input parameters of these cases are gathered. Besides, all the $\left(U_{i}, P_{i}\right)$ data pairs are back calculated from these obtained GRCs. Combining other input parameters, strength and softening parameters, with the back calculated $\left(U_{i}, P_{i}\right)$ data pairs, a total 547 numbers of data series are achieved considering all the studied cases. Based on the mentioned 547 gathered data series, support pressure is sorted as the function of 17 input parameters $P_{i}\left(r_{i}, G S I^{\text {peak }}, G S I^{\text {res }}, \sigma_{c i}, m^{\text {peak }}, s^{\text {peak }}, a^{\text {peak }}, m^{\text {res }}\right.$, $\left.s^{\text {res }}, a^{\text {res }}, E, \vartheta, \psi^{\text {peak }}, \psi^{\text {res }}, \sigma_{0}, \eta^{*}, U_{i}\right)$, where $m, s$ and $a$ represent Hoek-Brown constants of materials.

Then, using evolutionary polynomial regression modeling, the prepared data series are used to predict the available support pressure based on the input parameters and their relationship with the available support pressure.

\section{Sensitivity analysis}

In order to investigate the strength of relationship between input parameters and internal support pressure (to compare the degree of importance of each input parameter), the Cosine Amplitude Method (CAM) is considered. It should be noted that since $U_{i}$ was modeled as a fictional input parameter to model GRC, investigating its strength of relationship with internal support pressure as a variable does not mean and hence, here, its effect has not been investigated.

It should be noted that before carrying out the sensitivity analysis, all the input and output data are normalized and dimensionless in 0-1 scale using Eqn (9) (Ghorbani et al. 2014):

Scaled Value $=($ unscaled value - min. value $) /$ (max. value - min. value).

Thus, using CAM, the express similarity relation between the target function and the input parameters is attained. To do this, all of data pairs are expressed in the common $X$-space. They would form a data array $X$ defined as the Eqn (10) (Ghorbani et al. 2014):

$$
X=\left\{x_{1}, x_{2}, x_{3}, x_{4}, \ldots, x_{i}, \ldots x_{n}\right\},
$$

where each element, $x_{i}$, as shown in Eqn (11), is a vector of the length of $m$ (Ghorbani et al. 2014):

$$
x_{i}=\left\{x_{i 1}, x_{i 2}, x_{i 3}, \ldots, x_{i m}\right\} .
$$

Therefore, each of the datasets can be considered as a point in the $m$-dimensional space, where each point 
Table 4. The applied references for gathering strength parameters and deriving softening parameter used to model GRC based on HB and strain softening

\begin{tabular}{|c|c|}
\hline Hoek and Brown (1997) & In conjunction with Cai et al. (2007) and Alejano et al. (2010) \\
\hline Cai et al. (2007) & In conjunction with Hoek and Brown (1997) and Alejano et al. (2010) \\
\hline Lee and Pietruszczak (2008) & $\gamma_{i}^{p}=\gamma_{(i-1)}^{p}+\left(\Delta \varepsilon_{\theta(i)}^{p}-\Delta \varepsilon_{r(i)}^{p}\right)$ \\
\hline Park et al. (2008) & $\eta^{*}=(\alpha-1) \varepsilon_{\theta e}$ \\
\hline Zareifard and Fahimifar (2012) & $\begin{aligned} \gamma^{p} & =\varepsilon_{\theta}^{p}-\varepsilon_{r}^{p} \\
\dot{\varepsilon}_{r}^{p} & =\dot{\lambda} \frac{\partial g}{\partial \sigma_{r}} \\
\dot{\varepsilon}_{\theta}^{p} & =\dot{\lambda} \frac{\partial g}{\partial \sigma_{\theta}}\end{aligned}$ \\
\hline Alejano et al. (2010) & $\begin{array}{c}\varepsilon_{1}^{\text {plas }}=\left(\sigma_{1}^{\text {peak }}\left(\sigma_{3}\right)-\sigma_{1}^{\text {res }}\left(\sigma_{3}\right)\right)\left[\frac{1}{E}-\frac{1}{M}\right] \\
\varepsilon_{3}^{\text {plas }}=-\frac{1}{2} \frac{1+\sin \psi}{1-\sin \psi} \varepsilon_{1}^{\text {plas }} \\
\gamma^{p^{*}}=\eta^{*}=\varepsilon_{1}^{\text {plas }}-\varepsilon_{3}^{\text {plas }}\end{array}$ \\
\hline
\end{tabular}

requires $m$-coordinates to be fully described. Eqn (12) presents the strength of the relationship between $x_{i}$ and $x_{j}$ (Hasanzadehshooiili et al. 2012b; Ghorbani et al. 2014):

$$
r_{i j}=\frac{\sum_{k=1}^{m} x_{i k} x_{j k}}{\sqrt{\sum_{k=1}^{m} x_{i k}^{2} \sum_{k=1}^{m} x_{j k}^{2}}} .
$$

Figures 4 and 5, respectively, show the strength of relationship between input parameters and internal sup- port pressure for Mohr-Coulomb and Hoek-Brown strain softening cases.

As it can be seen, in-situ far field stress $\sigma_{0}, m^{\text {res }}$ and $\psi^{\text {res }}$ are the most affective parameters on the internal support pressure of tunnels in Hoek-Brown strain softening rock mass and peak and residual geological strength index of materials will more affect the support pressure of a strain softening Mohr-Coulomb rock mass. Besides, as it can be seen, $\gamma$ and $\vartheta$ are the least affective parameters on the GRC of strain softening MohrCoulomb material. Moreover, $a^{\text {res }}$ and $\eta^{*}$ have the least influence on the GRC of strain softening Hoek-Brown

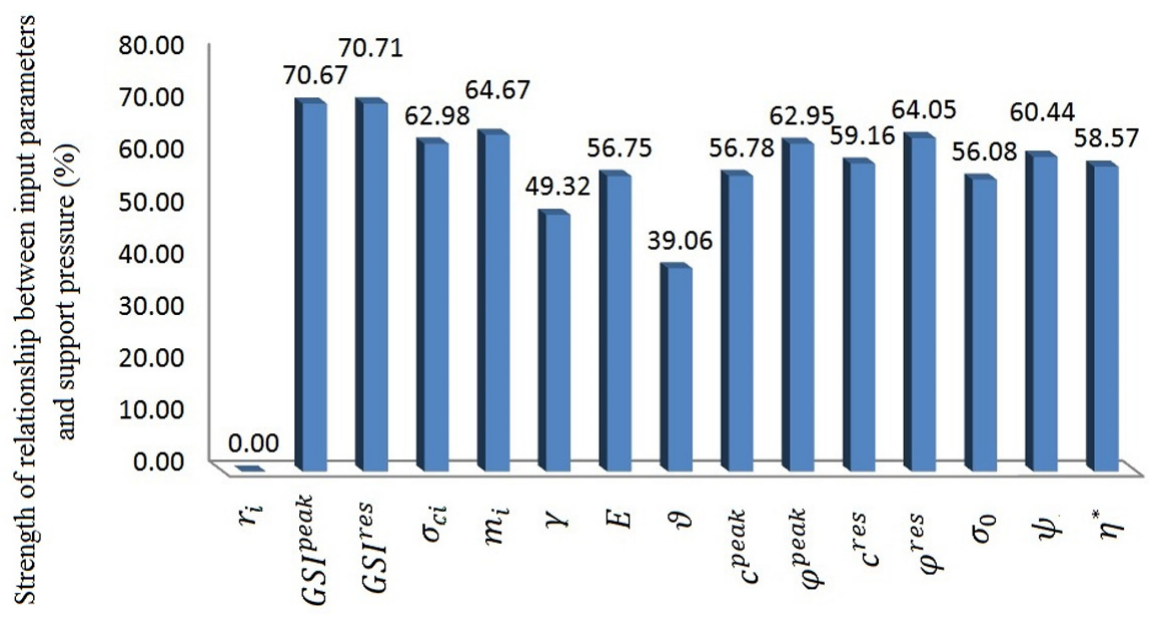

Fig. 4. Strength of relationship between internal support pressure and concerning parameters for the case of Mohr-Coulomb strain softening model 


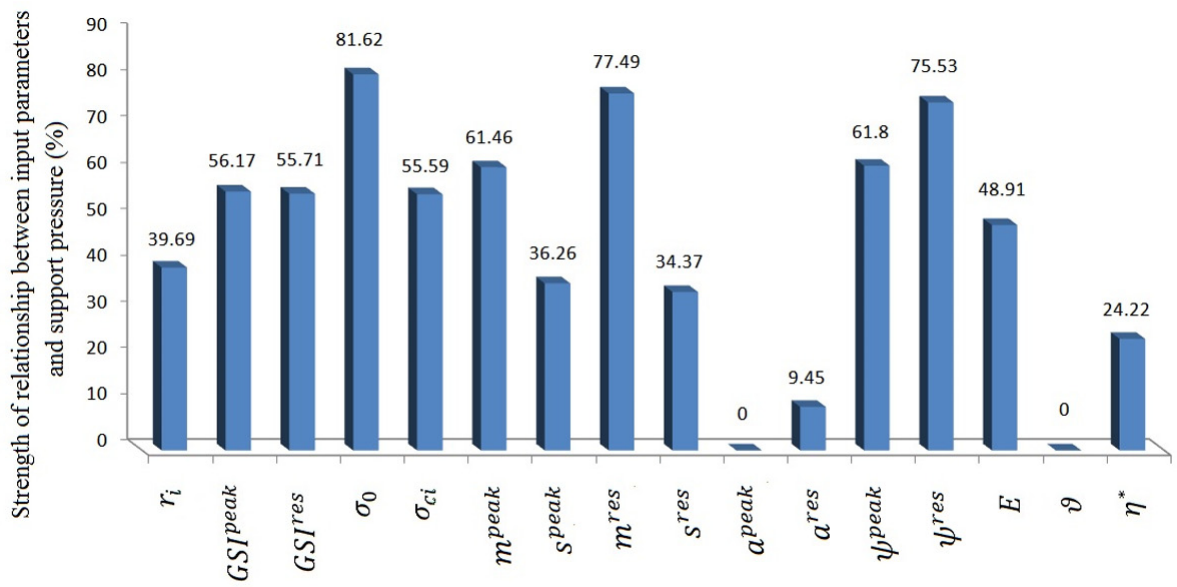

Fig. 5. Strength of relationship between internal support pressure and concerning parameters for the case of Hoek-Brown strain softening model

material. In addition, radius of tunnel in strain softening Mohr-Coulomb material, also, $a^{\text {peak }}$ and $\vartheta$ in HoekBrown strain softening material are constant input parameters in the development of all the studied ground reaction curves. Hence, the strength of relationship between these parameters and internal support pressure is reported as zero. Nevertheless, their values will vary in the case of studying larger databases, in which different $r_{i}, a^{\text {peak }}$ and $\vartheta$ values are available and used.

\section{Evolutionary polynomial regression modeling}

Different branches of artificial intelligence (AI) are applied to predict various complicated civil engineering functions (Baziar, Ghorbani 2005; Ghorbani et al. 2012; Sadrossadat et al. 2013; Sadowski, Nikoo 2014; Hasanzadehshooiili et al. 2014; Fiore et al. 2014; Gandomi et al. 2015; Sadowski, Hoła 2015; Yin et al. 2016; Turskis et al. 2016). Among the AI based modeling methods, EPR is classified as a symbolic grey box technique which can clearly make structured model expressions for a given dataset in a way that is shown in Figure 6. This figure

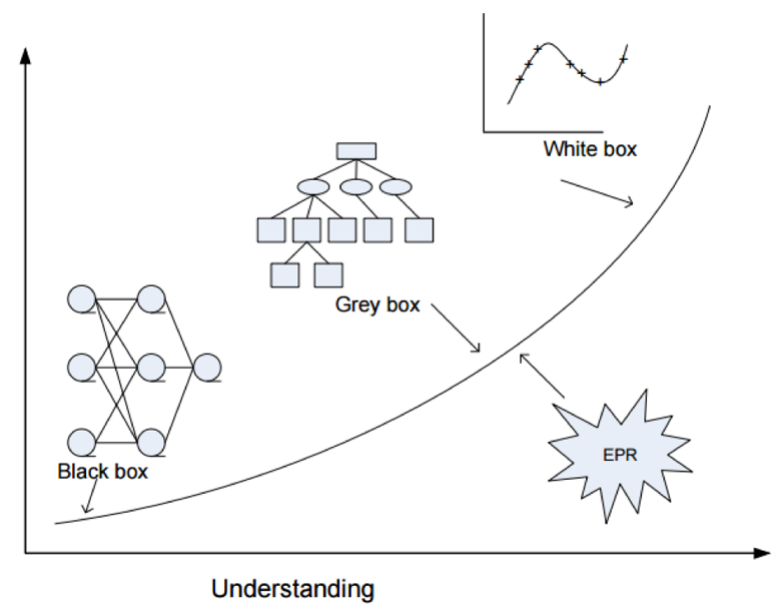

Fig. 6. EPR classification along with other modeling techniques (Rezania 2008) represents EPR classification along with other modeling methods (Rezania 2008).

EPR, which is a new hybrid regression method, combines the best features of conventional numerical regression techniques with the genetic programming symbolic regression technique. The main idea of this method is to employ an evolutionary computing methodology to search for a model of the system/process being modeled and to employ parameter estimation to obtain constants using least squares (Giustolisi, Savic 2006).

In genetic programming, a general evolutionary search is conducted in the evolutionary procedure while the exponents of a polynomial function with a fixed maximum number of terms are searched in EPR. Hence, the problem of mathematical expressions will be kept away from growing rapidly in length with time in evolutionary polynomial regressions. Moreover, selection of the optimum term numbers is permitted during one execution. Indeed, to do this, it returns some expressions with increasing numbers of terms up to a user defined threshold. The general expression form used in EPR is as Eqn (13) (Giustolisi, Savic 2006):

$$
y=\sum_{j=1}^{m} F\left(X, f(X), a_{j}\right)+a_{0},
$$

where $y$ represents the estimated vector of output, $a_{j}$ is a constant, $F$ is a function constructed by the process, $X$ is the matrix of input variables, $f$ is a user defined function and $m$ is the maximum term numbers of the target. Transferring Eqn (13) to the following vector form (Eqn (14)) is the first model identification step (Giustolisi, Savic 2006):

$$
\begin{aligned}
Y_{N \times 1}(\theta, Z)= & {\left[\begin{array}{ll}
I_{N \times 1} & Z_{N \times m}^{j}
\end{array}\right] \times\left[\begin{array}{llll}
a_{0} & a_{1} & \ldots & a_{m}
\end{array}\right]^{T}=} \\
& Z_{N \times d} \times \theta_{d \times 1}^{T}
\end{aligned}
$$

where $Y_{N \times 1}(\theta, Z)$ represents the least squares estimation vector of the $N$ target values, $\theta_{1 \times d}$ is the vector 
of $d=m+1$ parameters $a_{j}$ and $a_{0}$ (superscript " $T$ " represents the transposed vector), and $Z_{N \times d}$ is a matrix established by unitary vector for bias $a_{0}$, and $m$ vectors of variables $Z^{j}$ that for fixed $j$ are a product of the independent forecasters vectors of inputs, $X=\left\langle X_{1} X_{2} \ldots X_{K}\right\rangle$. Generally, construction of EPR models is formed in two stages. Firstly, the best function structure is realized using standard genetic algorithm and secondly, for each input combinations, the adjustable parameters are found conducting a least squares regression. Hence, a global search algorithm is put into the action for both the best set of input combinations and related exponents simultaneously, based on the cost function defined by the user. Eqn (15) presents matrix of inputs (Giustolisi, Savic 2009):

$$
X=\left[\begin{array}{ccccc}
x_{11} & x_{12} & x_{13} & \ldots & x_{1 k} \\
x_{21} & x_{22} & x_{23} & \ldots & x_{2 k} \\
x_{31} & x_{32} & x_{33} & \ldots & x_{3 k} \\
\ldots & \ldots & \ldots & \ldots & \ldots \\
x_{N 1} & x_{N 2} & x_{N 3} & \ldots & x_{N k}
\end{array}\right]=\left[\begin{array}{llll}
X_{1} & X_{2} & X_{3} & \ldots X_{K}
\end{array}\right]
$$

where $j^{\text {th }}$ term in Eqn (14) is represented by $k^{\text {th }}$ column of $X$ in Eqn (15). Hence, $j^{\text {th }}$ term of $Z$ in Eqn (14) will be re-written as Eqn (16) (Giustolisi, Savic 2009):

$$
\begin{aligned}
Z_{N \times 1}^{j}= & {\left[\left(X_{1}\right)^{E S(j, 1)} \cdot\left(X_{2}\right)^{E S(j, 2)} \cdot\left(X_{3}\right)^{E S(j, 3)} \ldots .\right.} \\
& \left.\left(X_{k}\right)^{E S(j, k)}\right] \forall j=1 \ldots m .
\end{aligned}
$$

In Eqn (16), $Z^{j}$ represents $j^{\text {th }}$ column vector and product of candidate independent inputs makes its elements. Also, ES is exponents' matrix. The final goal will be finding $E S_{m \times k}$ matrix of exponents in a way that its elements hold the values between user-defined limits. Then, based on the calculated $E S_{m \times k}$ matrix and using least square method (LS) and trying to minimize sum of squared errors (SSE) as the cost function, $a_{j}$ will be evaluated. The expression used in EPR as the typical flow diagram is shown in Figure 7 (Giustolisi, Savic 2009).

In the evolutionary process of building EPR models, a number of constraints can be implemented to control the output models in terms of the type of functions used,

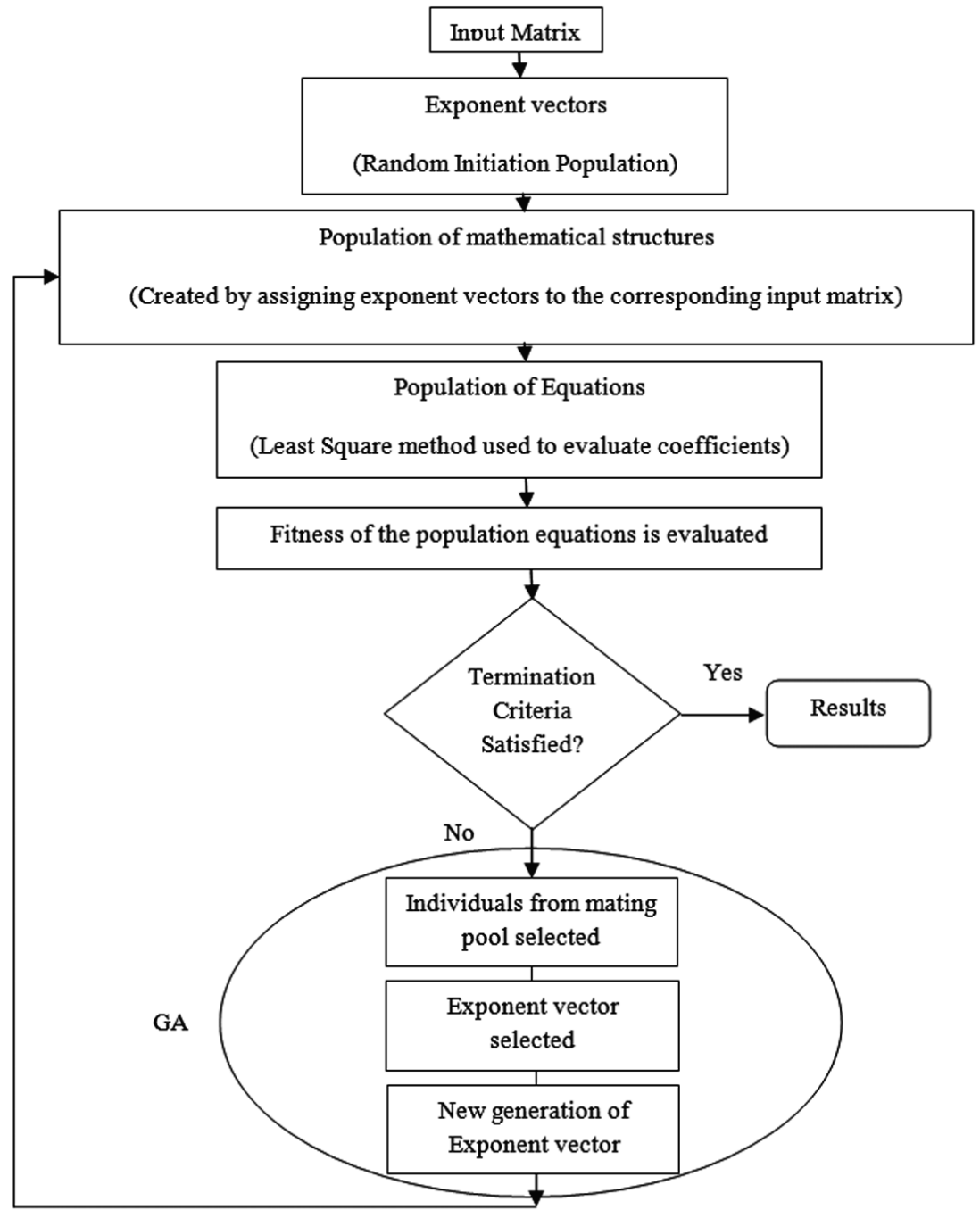

Fig. 7. Typical flow diagram for EPR procedure (Ahangar-Asr et al. 2011) 
number of terms, range of exponents, number of generations, etc. (Rezania et al. 2011). By increasing the number of evolutions, it gradually picks up different participating parameters in order to form equations describing the relationship between the parameters of the system. The level of accuracy of the output models is evaluated using the coefficient of determination. And, if the fitness of the model is not acceptable or the other termination criteria (in terms of maximum number of generations and maximum number of terms) are not satisfied, in order to gain a new model, the current model goes through another evolution (Ahangar-Asr et al. 2011).

\section{Results and discussion}

\subsection{EPR models based on Mohr-Coulomb strength criterion and strain softening}

As it was described in the previous sections, 16 input parameters (including $U_{i}$ ) is used to train and predict the values of $P_{i} \mathrm{~s}, P_{i}\left(r_{i}, G S I^{\text {peak }}, G S I^{\text {res }}, \sigma_{c i}, \gamma, E\right.$, $\left.\vartheta, c^{\text {peak }}, \varphi^{\text {peak }}, c^{\text {res }}, \varphi^{\text {res }}, m_{i}, \psi, \sigma_{0}, \eta^{*}, U_{i}\right)$.
Eqn (17) presents the relation obtained for predicting $P_{i}$ based on the input parameters:

$$
\begin{aligned}
& P_{i}=\left(4.4062 \times 10^{-7} \times e^{2 \psi}\right)+\left(5.9109 \times 10^{-11} \times e^{E} \times U_{i}\right)- \\
& \left(0.0015057 \times\left(\eta^{*}\right)^{2} e^{\sigma_{0}}\right)+\left(0.0032054 \times \vartheta \times\left(\varphi^{\text {res }}\right)^{0.5} \times U_{i}\right)- \\
& \left(0.00044979 \times\left(G S I^{\text {peak }}\right)^{0.5} \times \sigma_{c i} \times e^{\left(\vartheta+0.5 c^{\text {peak }}+c^{\text {res }}\right)} \times U_{i}^{0.5}\right)+
\end{aligned}
$$

5.7746 .

Figure 8 compares the predicted $P_{i}$ values (gained based on the new proposed equation) along with the calculated $P_{i}$ values (available in the authenticated literature for different number of datasets). In Eqn (17), the values of $U_{i}$ are based on mm. Also, $P_{i}$ values are calculated in $\mathrm{MPa}$.

Besides, Figure 9 shows the GRC points gained by EPR modeling versus their corresponding values available in the literature. As it can be seen, high value of coefficient of determination, $97.98 \%$, shows the high capability of new proposed model in prediction of GRC curve of circular tunnels in strain softening rock masses obeying Mohr-Coulomb criterion.

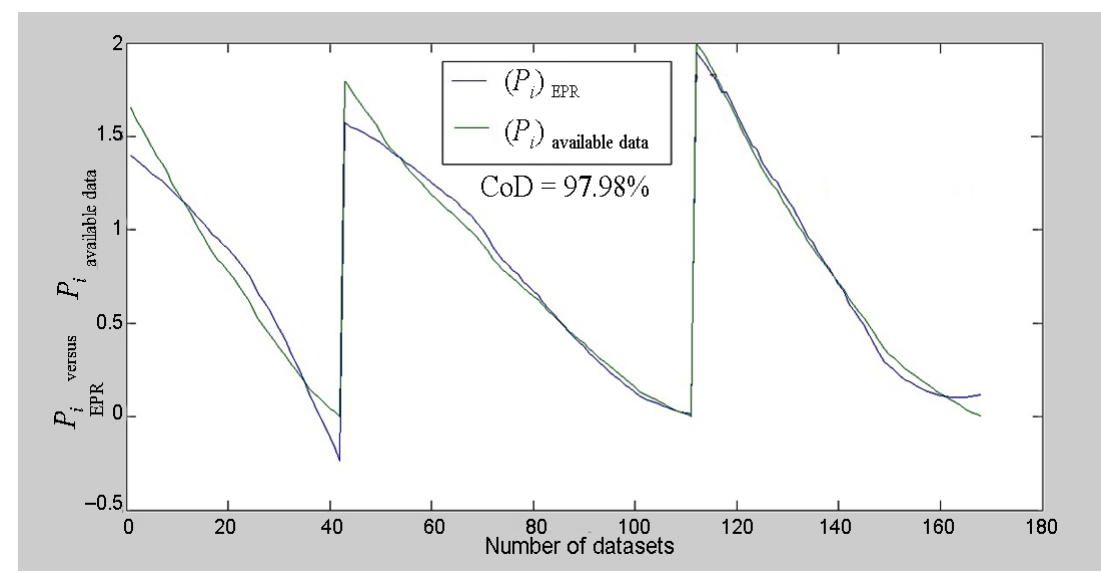

Fig. 8. Comparing the predicted $P_{i}$ with those available in the literature (based on Mohr-Coulomb strength criterion and strain softening)

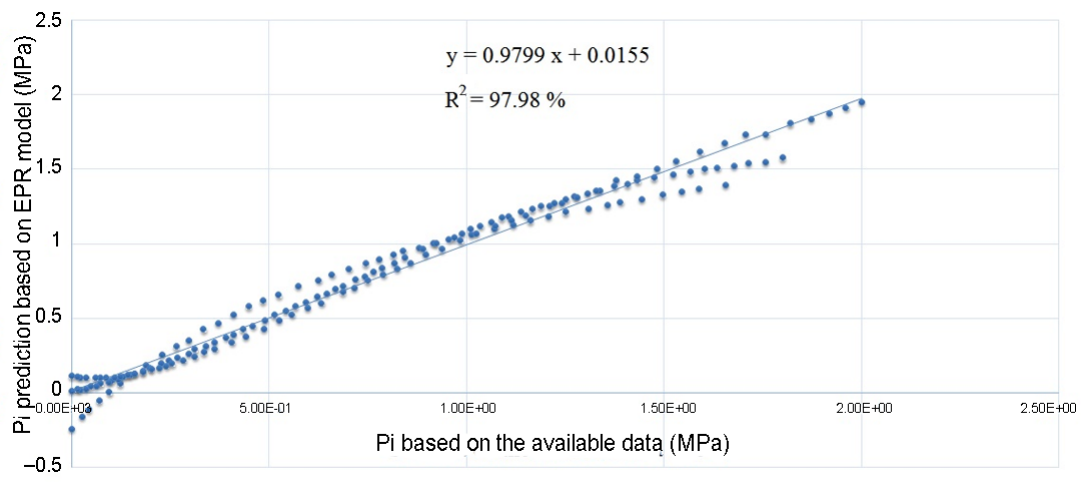

Fig. 9. Coefficient of determination for new proposed EPR-based GRC model for Mohr-Coulomb strength criterion and strain softening 


\subsubsection{Verification of developed EPR equation for Mohr-Coulomb criterion}

In order to evaluate the accuracy of the proposed equation, ground reaction curves available in the literature and used to develop EPR relation are used and the results gained from the new EPR equations are compared with those available in the literature.

Figure 10 presents EPR-proposed GRC versus their corresponding curves in the literature for three cases of Alejano et al. (2009), soft, medium and hard quality rock masses. As it can be seen, the proposed equation can be efficiently used as a first order estimation in preliminary design step of tunnels and can accurately predict the GRC of such cases.

\subsection{EPR models based on Hoek-Brown strength criterion and strain softening}

Similar to the previous section, the values of $P_{i}\left(r_{i}, G S I^{\text {peak }}, G S I^{\text {res }}, \sigma_{c i}, m^{\text {peak }}, s^{\text {peak }}, a^{\text {peak }}, m^{\text {res }}\right.$, $\left.s^{\text {res }}, a^{\text {res }}, E, \vartheta, \psi^{\text {peak }}, \psi^{\text {res }}, \sigma_{0}, \eta^{*}, U_{i}\right)$ are predicted using EPR modeling based on 547 used data sets.

Equation (18) represents the gained relation for $P_{i}$ (MPa) based on the concerning input parameters. In this equation, dimension of $U_{i}$ is meter:

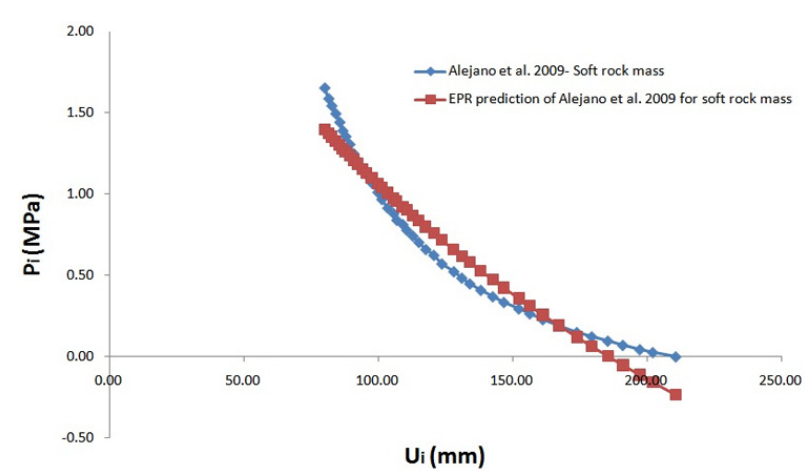

a) Alejano et al. (2009) versus EPR proposed curve of Alejano et al. (2009) case (soft rock mass)

$$
\begin{aligned}
& P_{i}=\left(1555.515 \times\left(a^{\text {peak }}\right)^{0.5} \times e^{\left(s^{\text {res }}+0.5 U_{i}\right)}\right)+ \\
& \left(33.5852 \times r_{i}^{0.5} \times e^{0.5 U_{i}} \times U_{i}^{0.5}\right)- \\
& \left(103.3917 \times r_{i}^{0.5} \times e^{\left(2 s^{\text {peak }}+0.5 m^{\text {res }}+2 U_{i}\right)} \times U_{i}^{0.5}\right)+ \\
& \left(7.3712 \times 10^{-5} \times r_{i}^{0.5} \times \sigma_{0}^{0.5} \times m^{\text {res }} \times\left(\eta^{*}\right)^{0.5} \times e^{\left(0.5 \sigma_{0}+U_{i}\right)}\right)- \\
& \left(0.00016622 \times r_{i} \times G S I^{\text {res }} \times\left(s^{\text {peak }}\right)^{0.5} \times E^{0.5} \times e^{0.5 \psi^{\text {res }}}\right)-
\end{aligned}
$$

1084.1708.

Figures 11 and 12 show the performance of the developed equation. As it can be clearly seen in the figures, high acceptable coefficient of correlation, $94.2 \%$, proves the efficiency and the applicability of newly proposed equation.

It should be noted that introducing new strength and softening parameters, both developed models can be efficiently used in the preliminary design step of circular tunnels in strain softening rock masses.

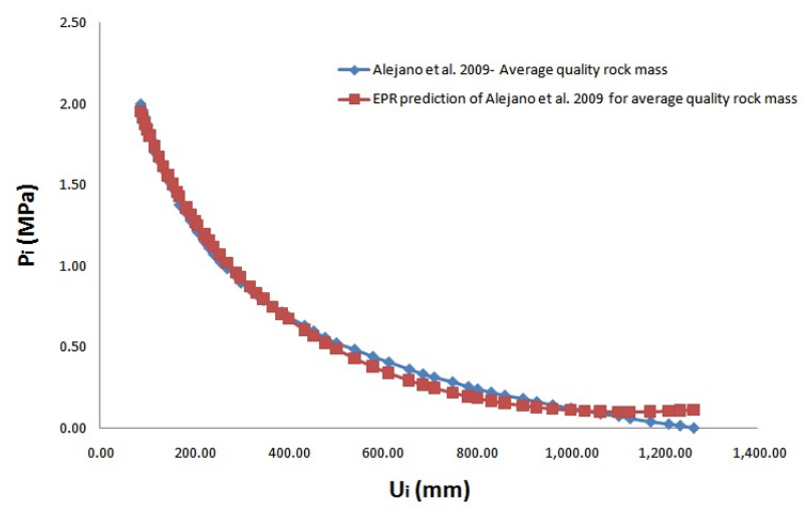

b) Alejano et al. (2009) versus EPR proposed curve of Alejano et al. (2009) case (medium rock mass)

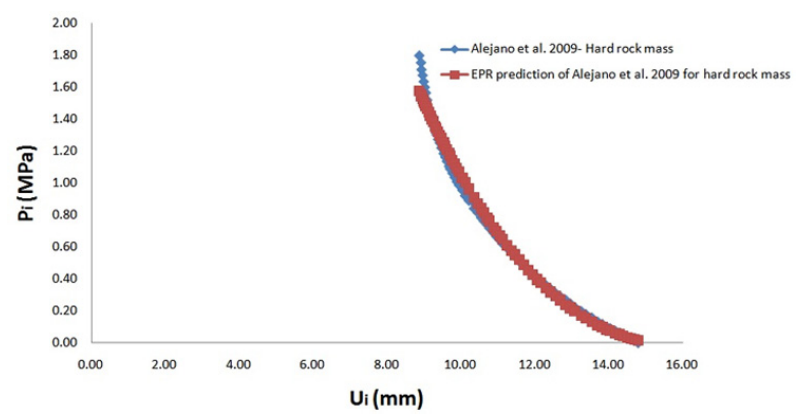

c) Alejano et al. (2009) versus EPR proposed curve of Alejano et al. (2009) case (hard rock mass)

Fig. 10. Comparison between available Mohr-Coulomb based GRC and their corresponding curves proposed by EPR 


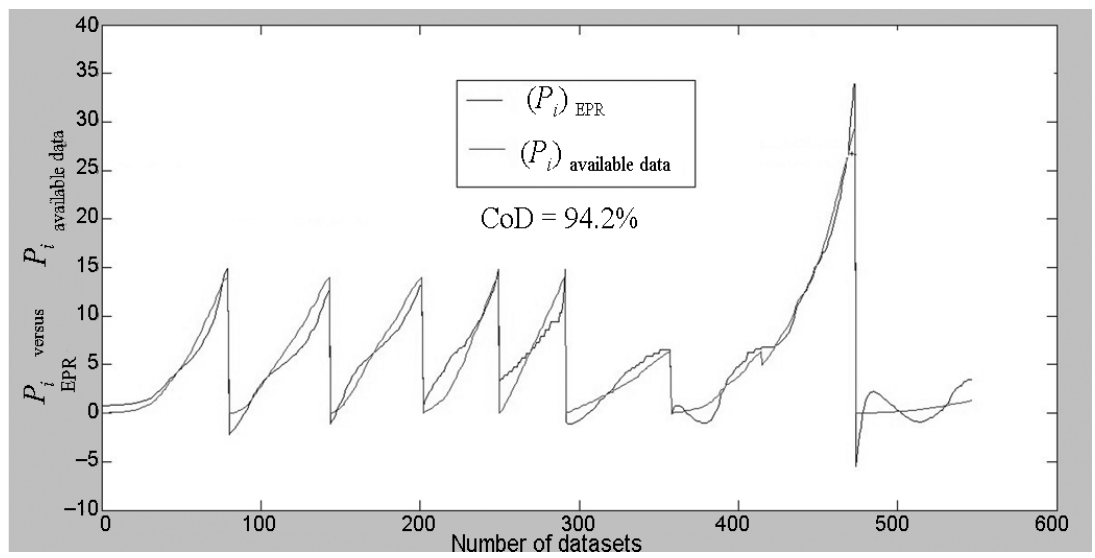

Fig. 11. Comparing the predicted with those available in the literature (based on Hoek-Brown strength criterion and strain softening)

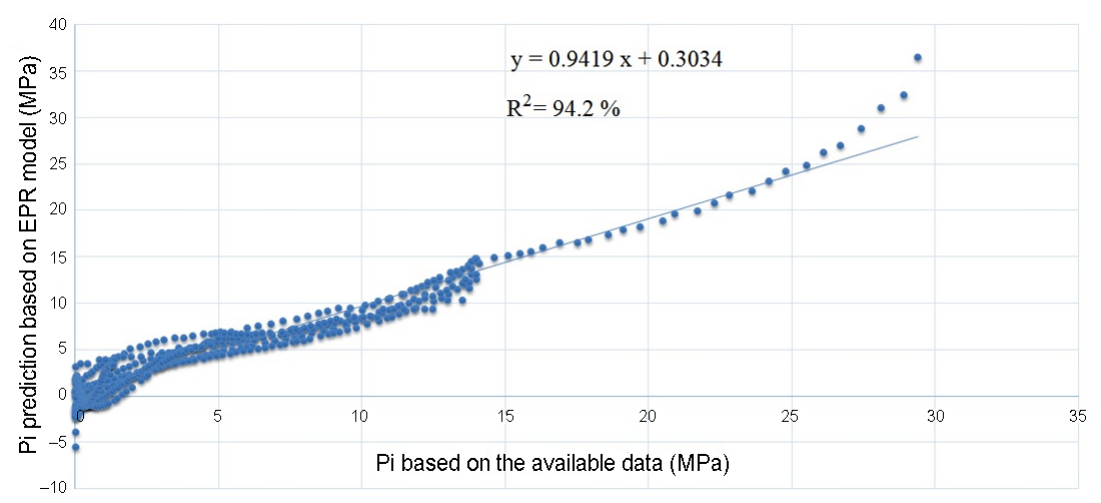

Fig. 12. Coefficient of determination for new proposed EPR-based GRC model for Hoek-Brown strength criterion and strain softening

\subsubsection{Verification of developed EPR equation for Hoek-Brown criterion}

In this section, accuracy of the proposed equation is examined through modeling five different cases available in the literature. In this regard, the corresponding GRC curves of all these five cases are predicted using the new proposed EPR equation and compared with those available in the literature. As it is shown in Figure 13, the acceptable prediction accuracy of new equation makes it useful for tunnel engineers and practitioners.

\section{Conclusions}

Because of difficulties in the development of realistic coupled theoretical-numerical modeling of GRC for strain softening rock masses, this papers aims to present an alternative simple novel model useful for practitioners and tunnel engineers to preliminary design the tunneling projects. In this regard, based on two well-known and widely used rock strength criteria, Mohr-Coulomb and Hoek-Brown failure criteria, two models for prediction of GRC of strain softening rock masses, believed to be more accurate, are presented based on the evolutionary poly- nomial regression modeling. The developed models are trained using available authenticated solutions for different quality strain softening rock masses and cover a wide range of rock mass qualities. Also, in order to investigate the role of strain softening of rock materials in GRC calculations, considering different plastic strain treatments, the parameter controlling softening of rock materials is also taken into account. It has been shown that the proposed equations are highly capable to predict the values of tunnel's internal support pressure based on rock masses strength and strain softening parameters. It is proved that the new proposed approach can be an efficient alternative for common available methods in the preliminary design steps of tunneling projects. Besides, based on the sensitivity analysis, the most and the least effective parameters on the values of tunnel's internal support pressure are introduced. Based on the CAM, in-situ stress, $m^{r e s}$ and $\psi^{r e s}$ are the most affective parameters on the support pressure of rock tunnels in Hoek-Brown strain softening rock mass and peak and residual geological strength index will more affect the support pressure of a strain softening Mohr-Coulomb rock mass. 


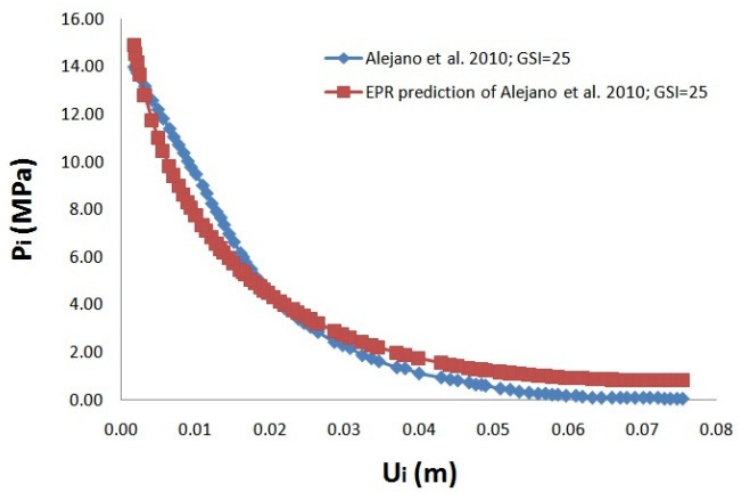

a) Alejano et al. (2010) versus EPR proposed curve of Alejano et al. (2010) case; GSI $=25$

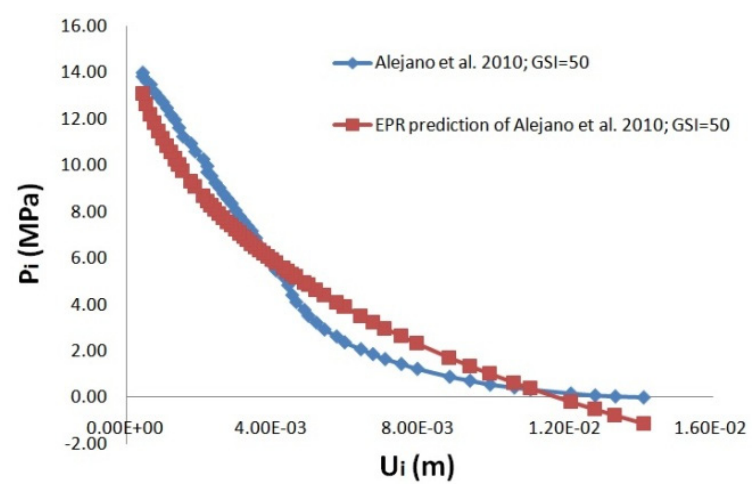

c) Alejano et al. (2010) versus EPR proposed curve of Alejano et al. (2010) case; GSI $=50$

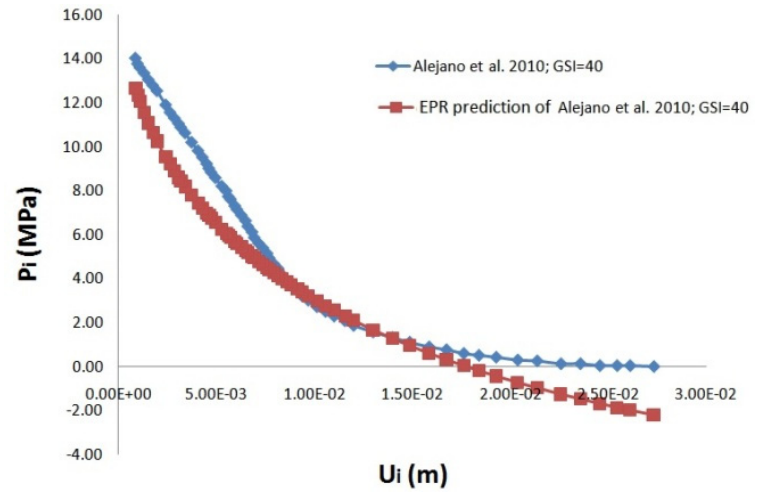

b) Alejano et al. (2010) versus EPR proposed curve of Alejano et al. (2010) case; GSI $=40$

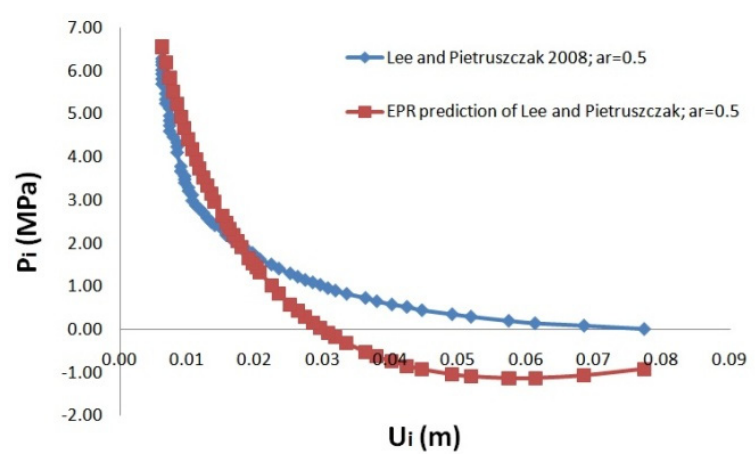

d) Lee and Pietruszczak (2008) versus EPR proposed curve of Lee and Pietruszczak (2008) case; $a_{r}=0.5$

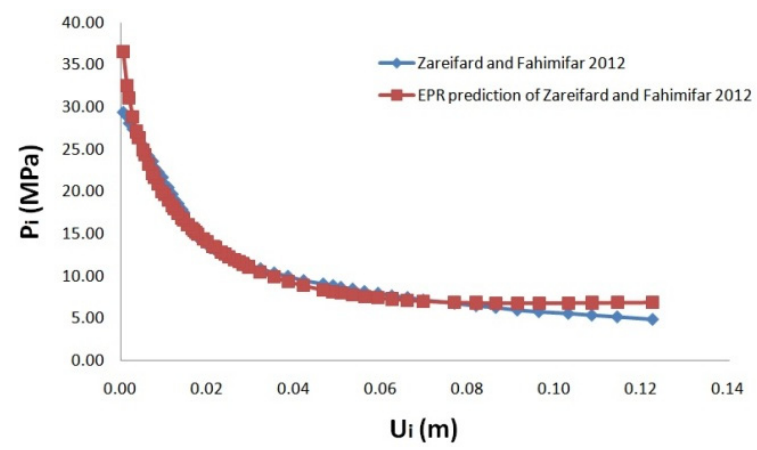

e) Zareifard and Fahimifar (2012) versus EPR proposed curve of Zareifard and Fahimifar (2012) case

Fig. 13. Comparison between some available Hoek-Brown based GRC and their corresponding curves proposed by EPR

\section{References}

Ahangar-Asr, A.; Faramarzi, A.; Mottaghifard, N.; Javadi, A. A. 2011. Modeling of permeability and compaction characteristics of soils using evolutionary polynomial regression, Computers \& Geosciences 37(11): 1860-1869. https://doi.org/10.1016/j.cageo.2011.04.015

Alejano, L. R.; Rodriguez-Dono, A.; Alonso, E.; Fdez-Manin, G. 2009. Ground reaction curves for tunnels excavated in different quality rock masses showing several types of post-failure behavior, Tunnelling and Underground Space Technology 24: 689-705.

https://doi.org/10.1016/j.tust.2009.07.004
Alejano, L. R.; Alonso, E.; Rodriguez-Dono, A.; Fdez-Manin, G. 2010. Application of the convergence-confinement method to tunnels in rock masses exhibiting Hoek-Brown strain-softening behavior, International Journal of Rock Mechanics \& Mining Sciences 47: 150-160. https://doi.org/10.1016/j.ijrmms.2009.07.008

Alonso, E.; Alejano, L. R.; Varas, F.; Fdez-Manin, G.; CarrenzaTorres, C. 2003. Ground response curves for rock masses exhibiting strain-softening behavior, International Journal for Numerical and Analytical Methods in Geomechanics 27: 1153-1185. https://doi.org/10.1002/nag.315 
Baziar, M. H.; Ghorbani, A. 2005. Evaluation of lateral spreading using artificial neural networks, Soil Dynamics and Earthquake Engineering 25(1): 1-9. https://doi.org/10.1016/j.soildyn.2004.09.001

Brown, E. T.; Bray, J. W.; Ladanyi, B.; Hoek, E. 1983. Ground response curves for rock tunnels, Journal of Geotechnical Engineering 109(1): 15-39.

https://doi.org/10.1061/(ASCE)0733-9410(1983)109:1(15)

Cai, M.; Kaiser, P. K.; Tasakab, Y.; Minamic, M. 2007. Determination of residual strength parameters of jointed rock masses using the GSI system, International Journal of Rock Mechanics \& Mining Sciences 44: 247-265. https://doi.org/10.1016/j.ijrmms.2006.07.005

Fahimifar, A.; Ranjbarnia, M. 2009. Analytical approach for the design of active grouted rock bolts in tunnel stability based on convergence-confinement method, Tunnelling and Underground Space Technology 24: 363-375. https://doi.org/10.1016/j.tust.2008.10.005

Fiore, A.; Quaranta, G.; Marano, G.; Monti, G. 2014. Evolutionary polynomial regression-based statistical determination of the shear capacity equation for reinforced concrete beams without stirrups, Journal of Computing in Civil Engineering, 04014111.

https://doi.org/10.1061/(ASCE)CP.1943-5487.0000450

Gandomi, A. H.; Faramarzifar, A.; Rezaee, P. G.; Asghari, A.; Talatahari, S. 2015. New design equations for elastic modulus of concrete using multi expression programming, Journal of Civil Engineering and Management 21(6): 761-774. https://doi.org/10.3846/13923730.2014.893910

Ghorbani, A.; Jafarian, Y.; Maghsoudi, M. S. 2012. Estimating shear wave velocity of soil deposits using polynomial neural networks: Application to liquefaction, Computers and Geosciences 44: 86-94. https://doi.org/10.1016/j.cageo.2012.03.002

Ghorbani, A.; Hasanzadehshooiili, H.; Shapalas, S.; Lakirouhani, A. 2013. Buckling of the steel liners of underground road structures: the sensitivity analysis of geometrical parameters, The Baltic Journal of Road and Bridge Engineering 8(4): 250-254. https://doi.org/10.3846/bjrbe.2013.32

Ghorbani, A.; Hasanzadehshooiili, H.; Ghamari, E.; Medzvieckas, J. 2014. Comprehensive three dimensional finite element analysis, parametric study and sensitivity analysis on the seismic performance of soil - micropile-superstructure interaction, Soil Dynamics and Earthquake Engineering 58: 21-36. https://doi.org/10.1016/j.soildyn.2013.12.001

Giustolisi, O.; Savic, D. A. 2006. A symbolic data-driven technique based on evolutionary polynomial regression, Journal of Hydroinformatics 8(3): 207-222.

Giustolisi, O.; Savic, D. A. 2009. Advances in data-drive analyses and modelling using EPR-MOGA, Journal of Hydroinformatics 11(3-4): 225-236.

https://doi.org/10.2166/hydro.2009.017

González-Cao, J.; Varas, F.; Bastante, F. G.; Alejano, L. R. 2013. Ground reaction curves for circular excavations in non-homogeneous, axisymmetric strain-softening rock masses, Journal of Rock Mechanics and Geotechnical Engineering 5: 431-442. https://doi.org/10.1016/j.jrmge.2013.08.001

Guan, Z.; Jiang, Y.; Tanabasi, Y. 2007. Ground reaction analyses in conventional tunnelling excavation, Tunnelling and Underground Space Technology 22: 230-237. https://doi.org/10.1016/j.tust.2006.06.004

Hasanzadehshooiili, H.; Lakirouhani, A.; Medzvieckas, J. 2012a. Evaluating elastic-plastic behaviour of rock materials using Hoek-Brown failure criterion, Journal of Civil Engineering and Management 18(3): 402-407. https://doi.org/10.3846/13923730.2012.693535
Hasanzadehshooiili, H.; Lakirouhani, A.; Medzvieckas, J. 2012 b. Superiority of artificial neural networks over statistical methods in prediction of the optimal length of rock bolts, Journal of Civil Engineering and Management 18(5): 655-661.

https://doi.org/10.3846/13923730.2012.724029

Hasanzadehshooiili, H.; Mahinroosta, R.; Lakirouhani, A.; Oshtaghi, V. 2014. Using artificial neural network (ANN) in prediction of collapse settlements of sandy gravels, Arabian Journal of Geosciences 7(6): 2303-2314.

https://doi.org/10.1007/s12517-013-0858-9

Hoek, E.; Brown, E. T. 1997. Practical estimates of rock mass strength, International Journal of Rock Mechanics and Mining Sciences 34(8): 1165-1186. https://doi.org/10.1016/S1365-1609(97)80069-X

Lee, Y. K.; Pietruszczak, S. 2008 A new numerical procedure for elasto-plastic analysis of a circular opening excavated in a strain-softening rock mass, Tunnelling and Underground Space Technology 23: 588-599. https://doi.org/10.1016/j.tust.2007.11.002

Mitaim, S.; Detournay, E. 2005. Determination of ground reaction curve for hyperbolic soil model using the hodograph method, Canadian Geotechnical Journal 42(3): 964-968. https://doi.org/10.1139/t05-024

Osgoui, R. 2009. Numerical-aided elasto-plastic model for circular tunnel in Hoek-Brown rock masses, in Proc. of ISRM International Symposium on Rock Mechanics: "Rock Characterization, Modelling and Engineering Design Methods" (SINOROCK2009), 19-22 May 2009, University of Hong Kong, China.

Park, K. H. 2014. Similarity solution for a spherical or circular opening in elastic-strain softening rock mass, International Journal of Rock Mechanics \& Mining Sciences 71: 151-159. https://doi.org/10.1016/j.ijrmms.2014.07.003

Park, K. H.; Tontavanich, B.; Lee, J. G. 2008. A simple procedure for ground response curve of circular tunnel in elastic-strain softening rock masses, Tunnelling and Underground Space Technology 23(2): 151-159. https://doi.org/10.1016/j.tust.2007.03.002

Rezania, M. 2008. Evolutionary polynomial pegression based constitutive modelling and incorporation in finite element analysis: PhD thesis. School of Engineering, Computing and Mathematics, University of Exeter. $172 \mathrm{p}$.

Rezania, M.; Javadi, A. A.; Giustolisi, O. 2011. An evolutionary-based data mining technique for assessment of civil engineering systems, Journal of Engineering Computations 25(6): 500-517. https://doi.org/10.1108/02644400810891526

Sadowski, L.; Nikoo, M. 2014. Corrosion current density prediction in reinforced concrete by imperialist competitive algorithm, Neural Computing and Applications 25(7): 1627-1638. https://doi.org/10.1007/s00521-014-1645-6

Sadowski, Ł.; Hoła, J. 2015. ANN modeling of pull-off adhesion of concrete layers, Advances in Engineering Software 89: 17-27. https://doi.org/10.1016/j.advengsoft.2015.06.013

Sadrossadat, E.; Soltani, F.; Mousavi, S. M.; Marandi, S. M.; Alavi, A. H. 2013. A new design equation for prediction of ultimate bearing capacity of shallow foundation on granular soils, Journal of Civil Engineering and Management 19(1): 578-590. https://doi.org/10.3846/13923730.2013.801902

Sayadi, A. R.; Lashgari, A.; Paraszczak, J. J. 2012. Hard-rock LHD cost estimation using single and multiple regressions based on principal component analysis, Tunnelling and Underground Space Technology 27(1): 133-141. https://doi.org/10.1016/j.tust.2011.08.006 
Sharan, S. K. 2003. Elastic-brittle-plastic analysis of circular openings in Hoek-Brown media, International Journal of Rock Mechanics and Mining Sciences 40(6): 817-24. https://doi.org/10.1016/S1365-1609(03)00040-6

Sharan, S. K. 2005. Exact and approximate solutions for displacements around circular openings in elastic-brittleplastic Hoek-Brown rock, International Journal of Rock Mechanics and Mining Sciences 42(4): 542-549. https://doi.org/10.1016/j.ijrmms.2005.03.019

Sharan, S. K. 2008. Analytical solutions for stresses and displacements around a circular opening in a generalized Hoek-Brown rock, International Journal of Rock Mechanics and Mining Sciences 45(1): 78-85. https://doi.org/10.1016/j.ijrmms.2007.03.002

Turskis, Z.; Daniūnas, A.; Zavadskas, E. K.; Medzvieckas, J. 2016. Multicriteria evaluation of building foundation alternatives, Computer-Aided Civil and Infrastructure Engineering 31(9): 717-729. https://doi.org/10.1111/mice.12202

Wang, S.; Yin, S. 2011. A closed-form solution for a spherical cavity in the elastic-brittle-plastic medium, Tunnelling and Underground Space Technology 26: 236-241. https://doi.org/10.1016/j.tust.2010.06.005

Xu, S. Q.; Yu, M. H. 2006. The effect of the intermediate principal stress on the ground response of circular openings in rock mass, Rock Mechanics and Rock Engineering 39(2): 169-181. https://doi.org/10.1007/s00603-005-0064-5
Yin, Z-Y.; Jin, Y-F.; Huang, H-W.; Shen, S-L. 2016. Evolutionary polynomial regression based modelling of clay compressibility using an enhanced hybrid real-coded genetic algorithm, Engineering Geology 210: 158-167. https://doi.org/10.1016/j.enggeo.2016.06.016

Zareifard, M. R.; Fahimifar, A. 2012. A new solution for shallow and deep tunnels by considering the gravitational loads, Acta Geotechnica Slovenica 2: 37-49.

Zavadskas, E. K.; Antuchevičienè, J.; Kapliński, O. 2015. Multi-criteria decision making in civil engineering. Part II applications, Engineering Structures and Technologies 7(4): $151-167$. https://doi.org/10.3846/2029882X.2016.1139664

Zhang, C. G.; Zhang, Q. H.; Zhao, J. H.; Xu, F.; Wu, C. Z. 2010. Unified analytical solutions for a circular opening based on non-linear unified failure criterion, Journal of Zhejiang University-SCIENCE A (Applied Physics \& Engineering) 11(2): 71-79.

Zhang, C.; Zhao, J.; Zhang, Q.; Hu, X. 2012. A new closedform solution for circular openings modeled by the Unified Strength Theory and radius-dependent Young's modulus, Computers and Geotechnics 42: 118-128. https://doi.org/10.1016/j.compgeo.2012.01.005

Zhang, L.; Wu, X.; Ding, L.; Skibniewski, M. J.; Lu, Y. 2016. Bim-Based Risk Identification System in tunnel construction, Journal of Civil Engineering and Management 22(4): 529-539. https://doi.org/10.3846/13923730.2015.1023348

Ali GHORBANI. PhD Associ. Prof. of Dept. of Civil Engineering, University of Guilan, Rasht, Guilan, Iran. A graduate of Engineering Faculty of University of Guilan, Rasht, Guilan, Iran (1989). He respectively earned his MSc and PhD both in geotechnical engineering at Iran University of Science and Technology, Tehran, Iran in 1997 and 2007. He is author of more than 20 journal papers and more than 50 conference papers in English and Farsi. Research interests: piled-rafts, soil dynamics, geotechnical earthquake engineering, liquefaction, lateral spreading, tunnel design, soil improvement and the application of AI techniques in civil engineering.

Hadi HASANZADEHSHOOIILI. PhD candidate of Dept. of Civil Engineering, Faculty of Engineering, Rasht, Guilan, Iran. A graduate of Mining Engineering of Urmia University, Urmia, Iran (2008). MSc of Geotechnical Engineering (2011) at University of Zanjan, Zanjan, Iran. He has published more than 10 journal and conference papers. Research interests: rock and geo-mechanics, tunnel engineering, constitutive modeling of geo-materials, computational plasticity and mechanics, Hoek-Brown plasticity, numerical modeling, geotechnical earthquake engineering, deep foundations and application of AI techniques in civil engineering. 